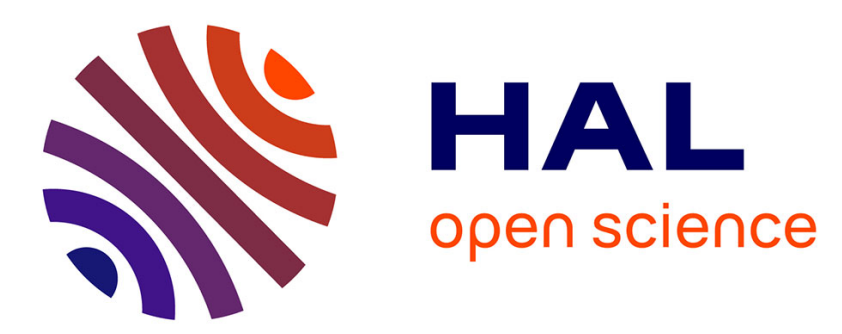

\title{
Etude par DRX et XPS des couches d'aluminium nitrurées par implantation ionique
}

Z. Seghrouchni, A. Mosser, J. Grob, N. Broll, A. Cornet

\section{To cite this version:}

Z. Seghrouchni, A. Mosser, J. Grob, N. Broll, A. Cornet. Etude par DRX et XPS des couches d'aluminium nitrurées par implantation ionique. Journal de Physique IV Proceedings, 1996, 06 (C4), pp.C4-501-C4-508. 10.1051/jp4:1996448 . jpa-00254331

\section{HAL Id: jpa-00254331 https://hal.science/jpa-00254331}

Submitted on 1 Jan 1996

HAL is a multi-disciplinary open access archive for the deposit and dissemination of scientific research documents, whether they are published or not. The documents may come from teaching and research institutions in France or abroad, or from public or private research centers.
L'archive ouverte pluridisciplinaire HAL, est destinée au dépôt et à la diffusion de documents scientifiques de niveau recherche, publiés ou non, émanant des établissements d'enseignement et de recherche français ou étrangers, des laboratoires publics ou privés. 


\title{
Etude par DRX et XPS des couches d'aluminium nitrurées par implantation ionique
}

\author{
Z. Seghrouchni, A. Mosser, J.J. Grob*, N. Broll** et A. Cornet** \\ IPCMS-GSI, 23 rue du Loess, 67037 Strasbourg cedex, France \\ * PHASE, 23 rue du Loess, 67037 Strasbourg cedex, France \\ ** LMCM-ENSAIS, 24 boulevard de la Victoire, 67000 Strasbourg cedex, France
}

Résumé: la majorité des travaux réalisés sur AN obtenu par implantation d'azote dans de l'aluminium a abouti à la formation de AIN de structure hcp. Cependant, sa structure cubique a été rarement observée. Une étude par diffraction des rayons $\mathrm{X}$, associée à la spectroscopie XPS, sur des films de $\mathrm{Al}(3000 \AA)$ implantés à $60 \mathrm{keV}$ avec des doses de $1.10^{17}, 3.10^{17}$ et 5 . $10^{17} \mathrm{~N}^{+} / \mathrm{cm}^{2}$, est présentée. L'effet le plus important obsevé sur les différents spectres DRX est le dédoublement des raies de diffraction de $\mathrm{Al}$ qui est plus marqué à la dose intermédiaire. Il révèle la présence de deux phases cubiques ayant $4,032 \AA$ et $4,062 \AA$ comme paramètres de maille. Du nitrure d'aluminium de structure cfc $(\mathrm{a}=7,938 \AA)$ est aussi observé à cette dose. Finallement, après implantation à $5.10^{17} \mathrm{~N}^{+} / \mathrm{cm}^{2}$ AIN de structure hexagonale apparaît suivant les directions (100) et (002). D'autre part, les analyses XPS montrent un déplacement chimique de l'aluminum dû à son environnement en azote pour les trois doses d'implantation.

\begin{abstract}
There are several reports wich show the spontaneous formation of hexagonal AIN by direct nitrogen ion bombardement of $\mathrm{Al}$ matrix or deposited layers. Nevertheless ambiguous data on the formation of a cubic solid solution by ion implantation exist.

The present paper mainly deals with grazing angle X-ray diffraction investigation of Al thin films $(3000 \AA)$ deposited on glass plates and nitrogen implanted at $60 \mathrm{keV}$ with $1.10^{17}, 3.10^{17}$ and $5.10^{17}$ ions $/ \mathrm{cm}^{2}$.The main diffraction effect observed is a splitting of $\mathrm{Al}$ diffraction maxima which is markedly observable at the intermediate dose and reveals presence of two facecentred cubic structures with $4.032 \AA$ and $4.062 \AA$ as lattice parameters. Another well crystallised cubic structure belonging to AlN was also detected at this implantation dose, which is not the case for the low dose. Finally at the high dose hep AIN structure appears with strong diffraction directions (100) and (002). Furthermore XPS analysis show a chemical shift of the $\mathrm{A} 2 \mathrm{p}$ photoelectron line dependent on its nitrogen environment for the three implanted samples.
\end{abstract}

\section{INTRODUCTION}

Le nitrure d'aluminium est un matériau isolant qui possède une grande stabilité thermique ainsi que d'excellentes propriétés mécaniques. C'est donc un matériau de base pour l'industrie microélectronique ( passivation des surfaces des semiconducteurs, transmission des ondes acoustiques de surface, matériaux piézoélectriques etc. ). La majorité des recherches réalisées sur la production de AIN par implantation ionique a abouti à la formation de AIN de structure hexagonale compacte [1-8]. Cependant, certains auteurs ont observé la formation de AIN de structure cfe [9]. De plus, les informations sur la formation d'une solution solide après irradiation d'un métal ( bombardement par des particules chargés, par des neutrons ou par des rayons $\mathrm{X}$ ) sont presque inexistantes dans la littérature.

Dans ce travail, une étude détaillée par diffraction des rayons $X$ en incidence rasante sur des couches d'aluminium, d'épaisseur $3000 \AA$, déposées sur des lames de verre puis implantées par des ions azote de $60 \mathrm{keV}$ avec différentes doses, est présentée. Elle consiste à suivre l'état structural de l'aluminium dans les premiers stades de sa nitruration jusqu'à la formation de AIN 
de structure hcp. D'autre part, nous avons réalisé une étude par spectroscopie des photoélectrons XPS en vue d'associer le déplacement chimique de l'aluminium à son état structural.

\section{Procédure expérimentale}

Des films d'aluminium d'épaisseur $3000 \AA$ ont été déposés sur des lames de verre dans un vide résiduel de $2.10^{-5}$ mbar. Immédiatement après le dépôt, ces échantillons ont été implantés par des ions azote, les doses choisies étant de $1.10^{17}, 3.10^{17}$ et $5.10^{17} \mathrm{~N}^{+} / \mathrm{cm}^{2}$. Le faisceau ionique a été balayé afin d'obtenir une distribution de concentration d'azote uniforme sur toute la surface implantée. La densité de courant ionique était de $1,6 \mu \mathrm{A} / \mathrm{cm}^{2}$. Les concentrations d'azote maximales calculées par le programme TRIM [10], pour chacune des doses mentionnées auparavant, sont égales respectivement à 0,$16 ; 0,50$ et à $0,82 \%$ at (figure 1 ). La profondeur moyenne des ions implantés à $60 \mathrm{keV}$ est de $1562 \AA$.

Pour déterminer la structure des différentes phases formées après implantation, nous avons utilisé la diffraction des rayons X (DRX) associée à la spectroscopie des photoélectrons. (XPS). Les mesures DRX ont été effectuées à l'aide d'un diffractomètre SIEMENS D5000, l'anode du tube à rayons $\mathrm{X}$ étant en $\mathrm{Cu}$. La technique utilisée est la diffraction en incidence rasante. Pour avoir des informations uniquement sur la couche de Al implantée nous avons utilisé un angle d'incidence rasant égal à $0,3^{\circ}$; sachant que sous cet angle $50 \%$ d'intensité diffractée provient d'une profondeur de $3000 \AA$; ainsi on a des informations sur toute la couche de Al implantée. Les analyses XPS ont été réalisées en employant un tube à anode d'aluminium $(\mathrm{AlK} \alpha / 1486,6 \mathrm{eV})$ opérant à $10 \mathrm{kV}$ séquentiellement avec un bombardement ionique de la surface des échantillons. L'énergie des ions $\mathrm{Ar}^{+}$utilisée pour éroder la surface des échantillons était de $4 \mathrm{keV}$.

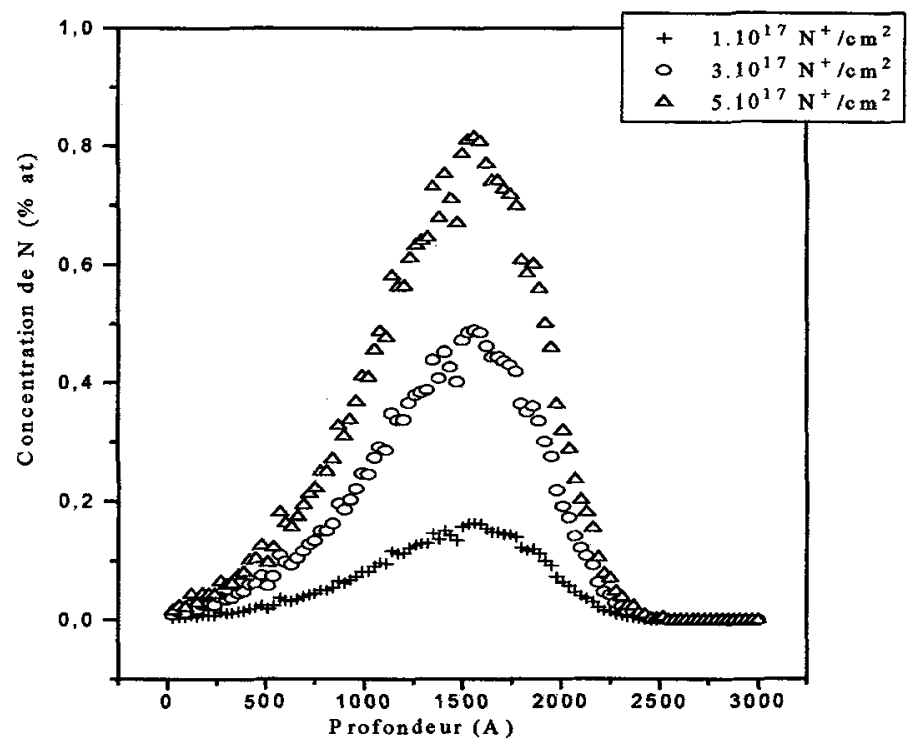

Figure1: Profil théorique, calculé par TRIM, des ions azote implantés à $60 \mathrm{keV}$ pour les les trois doses. The calculated profile of implanted nitrogen ions at $60 \mathrm{keV}$ for the three ion fases. 


\section{Résultats}

\subsection{Diffraction des rayons $X(D R X)$}

Les mesures de DRX effectuées sur les trois types d'échantillons implantés sont résumées dans la table1. Après implantation à $1.10^{17} \mathrm{~N}^{+} / \mathrm{cm}^{2}$, la raie $(200)$ de $\mathrm{Al}$ a une forme complexe qui laisse supposer qu'il y a superposition d'un maximum de faible contribution appartenant à une nouvelle phase cubique et du maximum, plus intense, de Al. En effet, en déconvoluant cette raie, nous trouvons un écart des paramètres de maille égal à $3,2 \cdot 10^{-2} \AA$. Le plus grand paramètre $(4,074 \AA)$ correspond à une nouvelle phase que nous attribuons à une solution solide Al-N, le deuxième $(4,042 \AA)$ correspond à la maille légèrement contractée de l'aluminium métallique $(4,059 \AA)$. Cette contraction peut être une conséquence des contraintes dues à l'insertion de l'azote dans la solution solide. La présence d'une phase de $\mathrm{Al}$ nitruré à côté de Al métallique est confirmée par les analyses XPS comme nous le verrons plus loin.

Table1: Paramètres de maille des différentes phases présentes dans les trois échantillons implantés.

: Lattice parameter of different phases obtained for the three implantation doses.

\begin{tabular}{|c|c|c|}
\hline $\begin{array}{c}\text { Doses, } \\
\mathrm{N}^{+} / \mathrm{cm}^{2}\end{array}$ & phases & $\begin{array}{c}\text { paramètres de } \\
\text { maille, } \AA\end{array}$ \\
\hline & $\mathrm{Al}$ & $\mathrm{a}=4,042$ \\
$1.10^{17}$ & $\mathrm{Al}-\mathrm{N}$ & $\mathrm{a}=4,074$ \\
\hline & $\mathrm{Al}$ & $\mathrm{a}=4,032$ \\
$3.10^{17}$ & $\mathrm{Al}-\mathrm{N}$ & $\mathrm{a}=4,062$ \\
& $\mathrm{AIN} \mathrm{cfc}$ & $\mathrm{a}=7,938$ \\
\hline & $\mathrm{Al}$ & $\mathrm{a}=4,047$ \\
$5.10^{17}$ & $\mathrm{Al}-\mathrm{N}$ & $\mathrm{a}=4,062$ \\
& $\mathrm{AIN} \mathrm{hcp}$ & $\mathrm{a}=3,141$ \\
& & $\mathrm{c}=4,992$ \\
\hline
\end{tabular}

Après implantation à $3.10^{17} \mathrm{~N}^{+} / \mathrm{cm}^{2}$, un dédoublement des raies de $\mathrm{Al}(111),(200)$ et $(220)$ est observé sur la figure 3. Nous pouvons plus aisément extraire les deux maximas correspondant à chacune des composantes de la raie. Comme précédemment, le maxima déplacé vers les angles de diffraction les plus élevés est attribué à $\mathrm{Al}$ avec un paramètre de maille moyen, mesuré suivant les trois directions cristallographiques, égal à $4,032 \AA$. Le deuxième, déplacé vers les petits angles, correspond à une phase cubique caractérisée par un écart moyen de paramètre de maille égal à $3,0.10^{-2} \AA$. Nous pouvons constater que cet écart est du même ordre de grandeur que celui mesuré pour la dose $1.10^{17} \mathrm{~N}^{+} / \mathrm{cm}^{2}$. La deuxième phase observée est alors attribuée à une solution solide Al-N. De plus, nous remarquons que le paramètre de maille de $\mathrm{Al}$ diminue encore plus qu'à faible dose et ceci du fait de l'augmentation des contraintes avec l'ajout des ions azote implantés. Par ailleurs les raies de diffraction observées à $45,93^{\circ}(\mathrm{d}=1,9743 \AA)$ et à $66,59^{\circ}(\mathrm{d}=1,4032 \AA)$ montrent la formation de AIN de structure $\mathrm{cfc}^{1}$ de paramètre de maille égal à $7,938 \AA$.

${ }^{1}$ Finalement, après implantation à $5.10^{17} \mathrm{~N}^{+} / \mathrm{cm}^{2}$, le nitrure d'aluminium de structure hexagonale apparait; il est confirmé par l'observation, sur la figure 2 , des raies $(100)(d=2,720$ $\AA)$ et $(002)(\mathrm{d}=2,496 \AA)$. Le rapport $\mathrm{c} / \mathrm{a}$ de $\mathrm{AIN}$ est égal à 1,59 ; il diffère légèrement du rapport théorique $(1,633)$ d'une structure wurtzite idéale suite à une distribution irrégulière des liaisons tétrahédriques [11]. Le dédoublement des raies de diffraction de $\mathrm{Al}$ disparait au profit de la restauration d'une forme complexe déjà observée à faible dose. Après déconvolution des

${ }^{1}$ [34-0679] fiche ASTM de AlN cfc 
raies (111) et (200) de $\mathrm{Al}$ nous obtenons deux composantes associées à deux phases cubiques caractérisées par un écart de paramètres de maille moyen $\left(1,5.10^{-2} \AA\right)$ plus faible que pour les autres doses. Cette diminution peut être interprétée par la relaxation des contraintes dues à la formation de la structure hop de AlN.

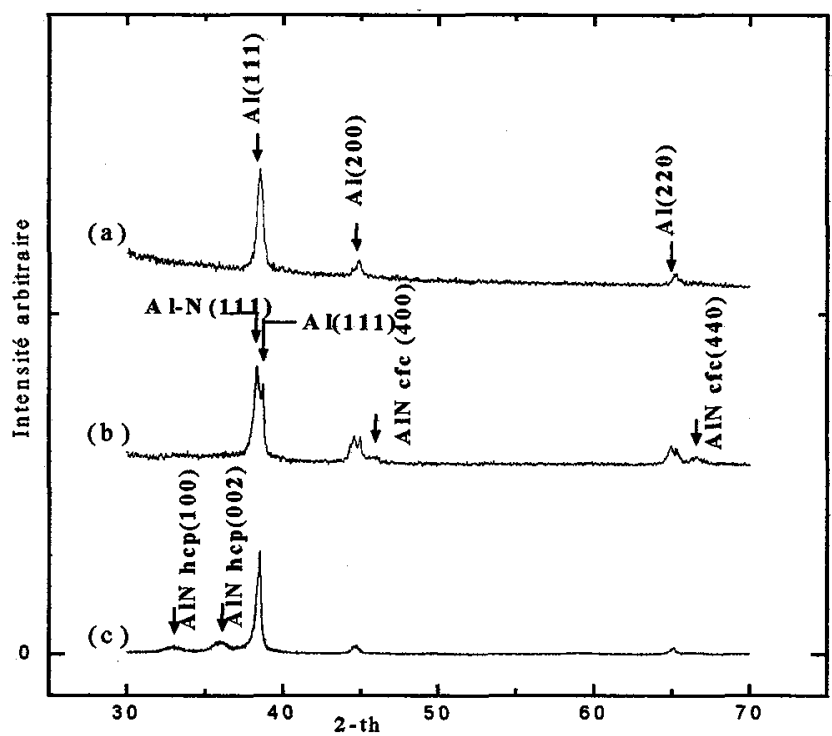

Figure 2 : Spectres de diffraction $X$ des films de $A 1$ implantés à (a) $1.10^{17} \mathrm{~N}^{+} / \mathrm{cm}^{2}$, (b) $3.10^{17} \mathrm{~N}^{+} / \mathrm{cm}^{2}$ et (c) $10^{17} \mathrm{~N}^{+} / \mathrm{cm}^{2}$.

: X-ray diffraction patterns for the aluminium specimens implanted with (a) $1.10^{17} \mathrm{~N}^{+} / \mathrm{cm}^{2}$, (b) 3 . $10^{17} \mathrm{~N}^{+} / \mathrm{cm}^{2}$ et (c) $5.10^{17} \mathrm{~N}^{+} / \mathrm{cm}^{2}$.
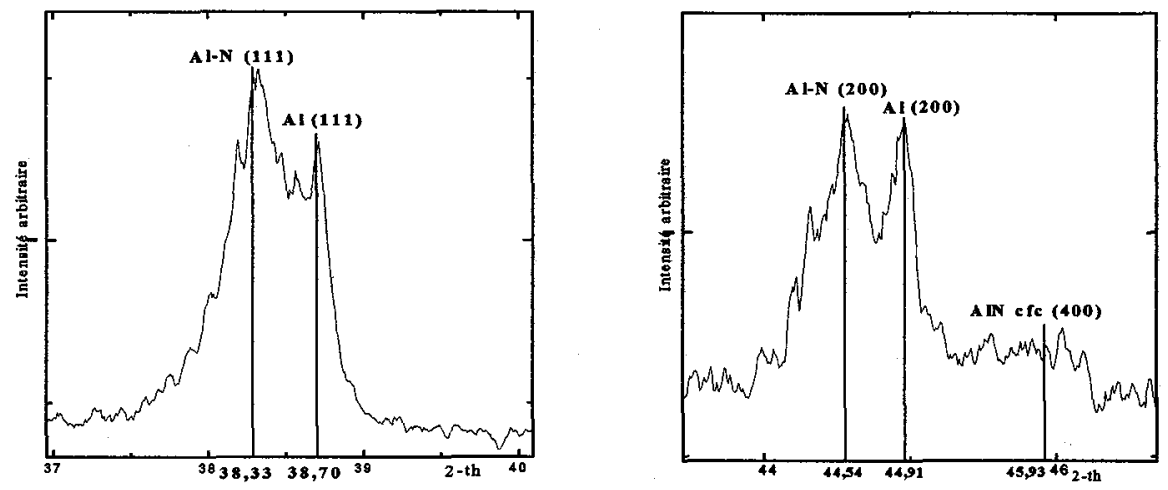


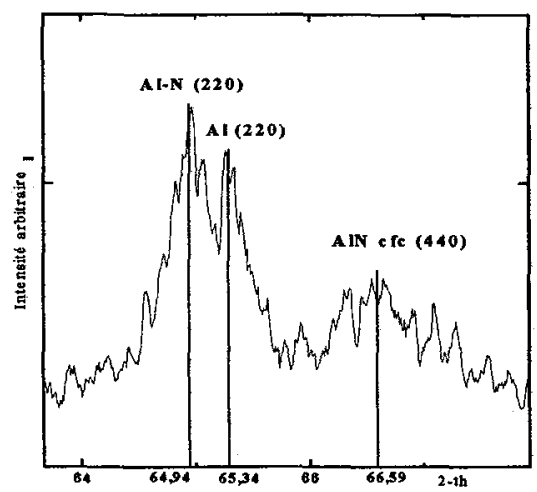

Figure 3 : Eclatement des raies de diffraction $(111),(200)$ et $(220)^{\circ}$ de $\mathrm{Al}$ implanté à $3.10^{17} \mathrm{~N}^{+} / \mathrm{cm}^{2}$. : Splitting of diffraction lines from Al implanted at $3.10^{17} \mathrm{~N}^{+} / \mathrm{cm}^{2}$.

\subsection{Spectroscopie des photoélectrons (XPS)}

Pour chaque type d'implantation, les analyses XPS ont été effectuées à différentes profondeurs, après érosion par bombardement ionique (figure 4). Avant bombardement ionique, l'extrême surface étant complètement oxydée, nous observons un pic d'aluminium caractéristique de l'alumine déplacé $(76,2 \mathrm{eV})$ par un effet de charge $(+2,6 \mathrm{eV})$ qui est mesuré par référence au pic C 1s. Nous notons que l'effet de charge est observé uniquement pour ce premier état de surface. La figure 5 montre le résultat de la déconvolution du pic Al $2 \mathrm{p}$ obtenu pour l'échantillon implanté à $5.10^{17} \mathrm{~N}^{+} / \mathrm{cm}^{2}$ après 10 minutes de bombardement ionique. On constate la présence de trois pics correspondant respectivement à $\mathrm{Al}$ métal $(71,09 \mathrm{eV}), \mathrm{Al}$ nitruré $(72,64 \mathrm{eV})$ et $\mathrm{Al}$ oxydé $(73,63 \mathrm{eV})$. Le déplacement chimique par rapport à $\mathrm{Al}$ métallique $(1,55 \mathrm{eV})$ de $\mathrm{Al}$ nitruré est en accord avec les données de la littérature [12,13].

L'énergie de liaison du pic $\mathrm{N}$ 1s dans AIN donnée par la littérature varie entre $396,5 \mathrm{eV}$ et $397,3 \mathrm{eV}$. Cette énergie, mesurée pour les trois échantillons implantés $(396,1 \mathrm{eV})$ est attribuée à l'azote de l'aluminium nitruré. Au cours du décapage de la surface de l'échantillon implanté à $5.10^{17} \mathrm{~N}^{+} / \mathrm{cm}^{2}$, la variation de l'intensité de $\mathrm{N} 1 \mathrm{~s}$, à partir de la surface vers le volume, présente deux maximums. L'un, obtenu après 46 minutes de bombardement, temps approximativement égal à la moitié du temps nécessaire pour le décapage de la couche totale de $\mathrm{Al}$, correspond au maximum du profil d'implantation calculé par le programme TRIM. Il coincide également avec la nitruration complète de $\mathrm{Al}$. Tandis que l'autre obtenu après 10 minutes de bombardement est dû à la migration d'azote vers la surface (figure 6). Des profils similaires ont été observés pour l'aluminium implanté à température ambiante et à $150^{\circ} \mathrm{C}[14,13]$ ainsi que pour d'autres métaux $[15,16]$.

\section{CONCLUSIONS}

- Dans les trois conditions d'implantation, il y a formation de deux phases cubiques ; l'une est attribuée à l'aluminium dont le paramètre de maille change en fonction de la dose utilisée, et la deuxième correspond à une solution solide Al-N de paramètre de maille plus grand que celui de Al.

- Après implantation à $3.10^{17} \mathrm{~N}^{+} / \mathrm{cm}^{2}$, du nitrure d'aluminium de structure cfc est observé.

- Il y a apparition de AIN de structure hep après implantation à $5.10^{17} \mathrm{~N}^{+} / \mathrm{cm}^{2}$.

- le profil en azote déduit des analyses XPS, obtenu après implantation à $5.10^{17} \mathrm{~N}^{+} / \mathrm{cm}^{2}$, présente deux maximas: l'un traduit la migration d'azote vers la surface, l'autre, situé approximativement au milieu de la couche de $\mathrm{Al}$, correspond au maximum calculé par le programme TRIM à $60 \mathrm{keV}$. 
(a)
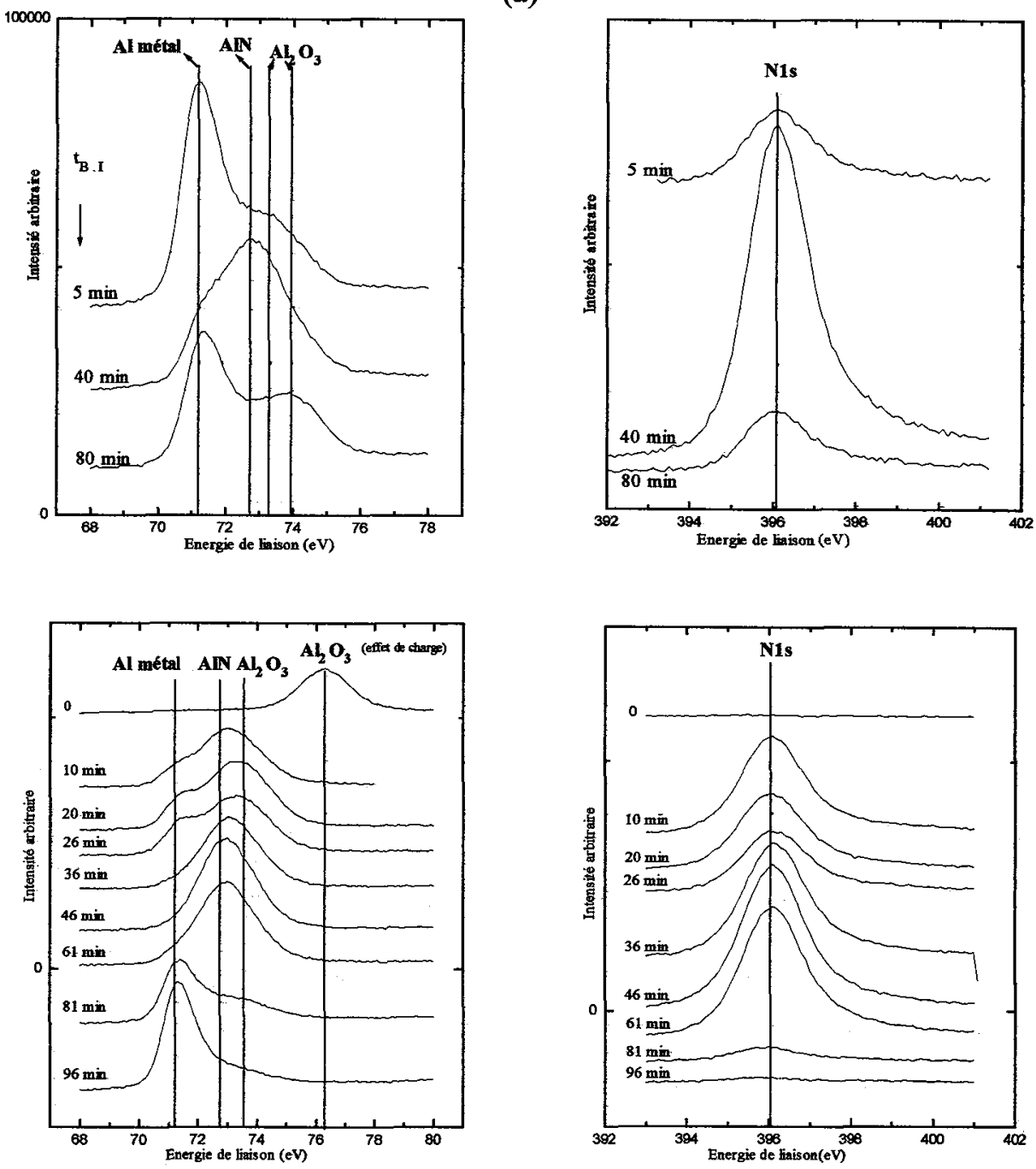

(b)

Fìgure 4 : Spectres XPS Al2p et N1s obtenus pour Al implanté avec (a) $1.10^{17} \mathrm{~N}^{+} / \mathrm{cm}^{2}$ et (b) $5.10^{17} \mathrm{~N}^{+} / \mathrm{cm}^{2}$, après différents temps de bombardement par les ions argon.

Al $2 p$ photoelectron spectrum obtained from $\mathrm{Al}$ after nitrogen ion implantation to a fluence of (a) $1.10^{17} \mathrm{~N}^{+} / \mathrm{cm}^{2}$ and (b) $5.10^{17} \mathrm{~N}^{+} / \mathrm{cm}^{2}$, after different intervals of argon ion etching. 
Par contre, ces analyses ne permettent pas, dans les conditions opératoires utilisées, de diffërencier les phases nitrurées Al-N, AIN cfc et AIN hcp.

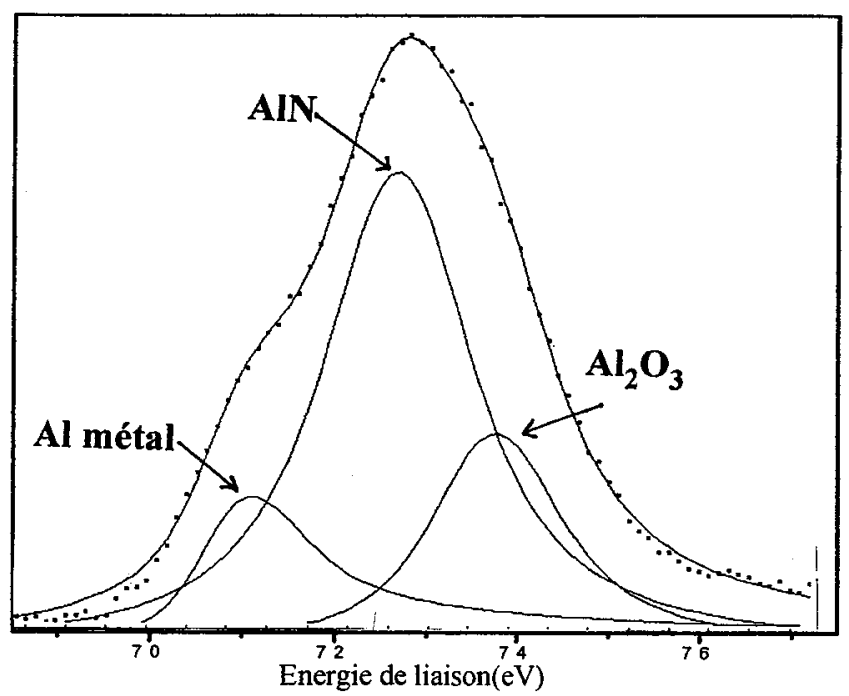

Figure 5 : Déconvolution du pic des photoélectrons $\mathrm{Al} 2 \mathrm{p}$ correspondant à la dose d'implantation $5.10^{17}$ $\mathrm{N}^{\mathrm{T}} / \mathrm{cm}^{2}$, après 10 minutes de bombardement ionique.

Deconvolution of Al 2p photoelectron peak from Al implanted at $5.10^{17} \mathrm{~N}^{+} / \mathrm{cm}^{2}$ spectrum obtained after an etching time of 10 minutes.

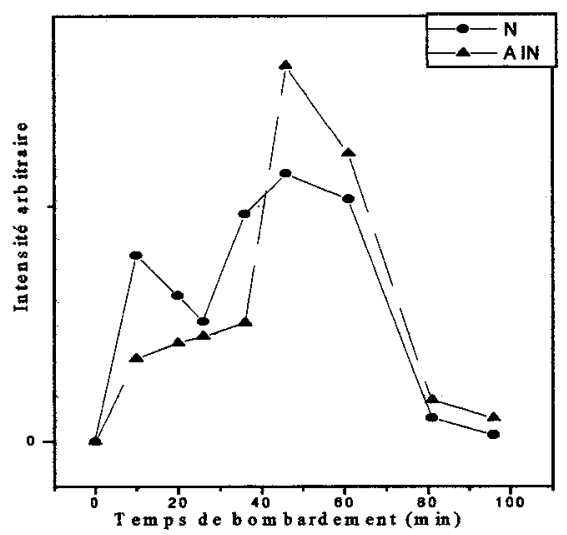

Figure 6 : Variation de l'intensité des pics (après soustraction du fond continu) de photoélectrons AlN2p et N1s en fonction du temps de bombardement ionique après implantation à $5.10^{17} \mathrm{~N}^{7} / \mathrm{cm}^{2}$.

Variation of the AlN2p and N1s photoelectron peak intensities (after background subtraction) with the sputtering time for the sample implanted with $5.10^{11} \mathrm{~N}^{+} / \mathrm{cm}^{2}$. 


\section{Références}

[1] Pavlov P.V., Zorin E.J.,.Tetelbamn P.I, Lesnikov V.P., Ryzhkov G.M. and Pavlov A.V., Phys. Status Solidi A, 19(1973)373.

[2]_Belyi I.M., Komarov F.F., Tishkov V.S. and Yankovskii V.M., Phys. Status Solidi A, 45(1978)343.

[3] Murayama Y. and Kashiwagi K. J. Vac. Sci. Technol., 17(1980)796.

[4] Morita M., Isogai S., Tsubouchi K. and Mikoshiba N., Appl. Phys. Lett., 38(1981)50.

[5] Kawabata A., Jpn. J. Appl. Phys.,23, Suppl.1, Part1(1984)17.

[6] Rauschenbach B., Kolitsch A. and.Richter E, Thin Solid Films, 109(1983)37.

[7] Ohira S.,. Iwaki M, Mater. Sci. Eng., 90(1987)143.

[8] Gautier M, Duraud J.P and LeGressus, J. Appl. Phys., 61(1987)574.

[9] A.P. Matthews, M.Iwaki, Y.Horino, M.Satou and K. Yabe, Nucl. Instrum .Methods Phys. Res., B59/60(1991)671.

[10] J.F.Ziegler, J.P. Biersack and U. Littmark, in: The Stopping and Ranges of Ions in Solids, vol.1, (ed.J.F.Ziegler, Pergamon, New York, 1985).

[11] Jeffrey G.A. and Parry G,S,. J. Chem. Phys., 23(1955)406.

Jeffrey G.A., Parry G,S and Mozzi R.L., J. Chem. Phys., $25(1956) 1024$.

[12] Taylor J.and Rabalais J.W., J. Chem. Phys., 75(1981)1735.

[13] Rauschenbach B., Breuer K. and Leonhardt G., Nucl. Instrum .Methods Phys. Res., B47(1990)396.

[14] Ohira S. and Iwaki M., Nucl. Instrum .Methods Phys. Res., B19/20(1987)162.

[15] Rauschenbach B., Nucl. Instrum .Methods Phys. Res., B15(1986)756.

[16] Piette M., Terwagne G., Möller W. and Bodart F., Mater. Sci. Eng., B2(1989)189 